\title{
Detent Force Reduction by Positional Shifting of Permanent Magnets for a PMLSM Without Compromising Thrust
}

\author{
Yuto Mitsui* \\ Non-member, \\ Yasuaki Aoyama** Member, \\ Salman Ahmed ${ }^{* a)}$ Student Member \\ Takafumi Koseki* Member
}

(Manuscript received July 4, 2019, revised Feb. 13, 2020)

\begin{abstract}
Detent force is caused by the interaction of a permanent magnet's flux with an armature's teeth even in the absence of armature excitation. It produces unwanted vibrations and hinders precise motion control of Permanent Magnet Linear Synchronous Motor (PMLSM). Reduction of detent force is still a challenge as it is always accompanied by reduction in the desired thrust. In this paper, a method to reduce the detent force without compromising thrust is proposed. By adjusting the position of the mover magnet with simultaneous re-arrangement of coil phases, significant reduction in the detent is achieved with negligible effect on thrust. Finally, simulation and prototype measurements were used to depict the usefulness of the proposed method.
\end{abstract}

Keywords: Permanent Magnet Linear Synchronous Motor (PMLSM), detent force reduction, high thrust density, longitudinal and transverse magnetic flux, phase shift, Harmonic analysis

\section{Introduction}

Permanent Magnet Linear Synchronous Motors (PMLSMs) are widely used in the industrial fields such as semiconductor manufacturing equipment and machine tools ${ }^{(1)}$. For these applications, PMLSM is generally required to have the following characteristics:

(1) High thrust density

(2) Low detent (Cogging) force

(3) Simple and easy to manufacture

Due to limitation of operating space, high thrust density is essential for up-sizing of objects in semiconductor manufacturing equipment and machine tools. In addition, high speed and high acceleration of PMLSM leads to reduced operation times in transportation, processing and inspection, which leads to higher productivity. Positional accuracy must be maintained at such high accelerations. However, employing large permanent magnets (PM) for high thrust is generally accompanied by a high detent force. It is the longitudinal component of attractive force between PM and iron core of armature, and exists even at no armature excitation. Not only does it decrease positional accuracy, but also leads to highspeed vibration due to its periodic nature.

The detent force can be classified into two types: slot effect and end effect. The slot effect, commonly known as cogging force in the rotary counterpart, is due to the interaction of the motor slots and $\mathrm{PM}^{(2)}$. Many methods have been proposed

\footnotetext{
a) Correspondence to: Salman Ahmed. E-mail: s.ahmed@koseki.t. u-tokyo.ac.jp

* Graduate School of Engineering, Dept. of Electrical Engineering and Information Systems, The University of Tokyo

7-3-1, Hongo, Bunkyo-ku, Tokyo 113-8656, Japan

** Research and Development Group, Hitachi, ltd Ibaraki, Japan
}

to reduce slot effects, such as skew adjustment, optimization of slot opening width and magnet thickness, and slot and pole combinations ${ }^{(3)-(6)}$. A slot pole combination having a high Least Common Multiple (LCM) ${ }^{(3)(4)}$ is sufficient to drastically reduce the slot effect while maintaining thrust large. However, despite low slot effect, linear motors also suffer from the end effect which is a consequence of finite length of the armature. In literature, we find many works dedicated to end effect reduction. Fixed on either sides, core-like structures (without windings), called Auxiliary Poles (AP), used to destructively eliminate end effects are investigated in (7)(9). However, adopting the auxiliary pole increases length of the armature and shortens the motor stroke. In addition, since the analytical evaluation of magnetic flux for AP has not been established, finding the optimal size and position is computationally an expensive task as one has to resort to Finite Element Method (FEM) for detent calculation ${ }^{(8)}$. Optimization of the armature length to destructively cancel out the end effect of both ends is also effective in reducing detent $^{(10)(11)}$, but this can result in asymmetry, which may distort the induced voltage, resulting in a thrust ripple in electromagnetic thrust. A new procedure which includes shaping the end core faces with two steps is proposed in (12). It is an extension of armature length optimization but in this case, up to two harmonics can be eliminated, however, it requires that experimental detent waveform of one side be known priorly. Moreover, a sectioning method was proposed in (13) which divided the motor into three parts allowing extra degree of freedom to eliminate up-to two end effect harmonics without reducing thrust, but, at the expense of increased volume.

In light of the merits and demerits of above mentioned methods, we propose a detent force reduction method applicable to double sided PMLSM, without compromising thrust or volume. A phase difference of $90 \mathrm{deg}$ is introduced 
between the two PM mover sides which eliminates the fundamental end effect harmonic. Conventionally for double sided structures, this is usually accompanied by a drastic reduction in thrust and is normally avoided. However, owing to the magnetic flux path being three dimensional, we are able to maintain the original thrust by rearrangement of armature winding. Although, phase shifting is common in literature works, it requires use of two or three independent motors, which demands accurate in-between positioning and also results in internal moments. In our machine, this is not the case since the armature is a single core, which adds ruggedness to the machine apart from ease of manufacturing. The internal moments are comparatively less and the application of thrust is collocated.

By application on motors of varying slot-pole combination, the usefulness and practical feasibility of the proposed method is verified. The paper is divided into five sections. Section 2 introduces the motor under consideration. In section 3, first, an analytical proof is provided, followed by phase reassignment strategy and a general formulation for applicable structures. The method is applied to different poleslot configured structures in later part of section 3. Section 4 deals with manufacturing of prototype and experimental validation of detent reduction and thrust retainment. Finally, the paper is concluded in section 5

\section{Hybrid Transverse and Longitudinal Flux Two-mover PMLSM}

The motor under consideration is a PMLSM with transverse and longitudinal magnetic fluxes as shown in Fig. ${ }^{(14)}$. Armature, which is the stator in this case, is a double sided structure enclosing the two permanent magnet (PM) movers. Transverse flux in the $y z$-plane is facilitated by iron structures, named "arm", connecting upper and lower sides ${ }^{\dagger}$. Whereas the longitudinal flux flows in the $x z$-plane, shown in the magnified cut view in Fig. 1(b), is facilitated by structure named "bridge" which connects the adjacent cores.

In this paper, the pole-slot combination used is $6 \tau_{S}=7 \tau_{P}$, where $\tau_{P}$ is the pole pitch and $\tau_{S}$ is the slot pitch. Each slot has a pair of copper coils amounting to a total of six pairs for each side (upper and lower). These when excited by three phase current produce thrust along the $x$-axis. In fact, the structure looks like having two PMLSMs stacked vertically along $z$-axis. By such assembly, the total volume of the armature is reduced since the both, upper and lower sides share a common in-between section. As will be shown in next section, this in-between section allows a bypass to magnetic flux when the phase between upper and lower PM sides is changed. Specifications of permanent magnets and armature are shown in the Table 1.

Furthermore, since the magnetic return paths are both transverse and longitudinal, the magnetic utilization factor is high which leads to large thrust density. Since armature core's laminations are stacked along $x$-axis, and the magnetic reluctance in transverse flux is lower than that in longitudinal direction. The transverse magnetic path is first utilized, and once saturated which increases the magnetic reluctance, the

\footnotetext{
$\dagger$ For the sake of consistency in the entire paper, all discussions refer to the two PM sides as upper and lower ones and all figures have been aligned accordingly.
}

Table 1. Specification of prototype

\begin{tabular}{l|l|c}
\hline & Material & $\mathrm{N} 48 \mathrm{H}$ \\
Magnet (Mover) & Remanence [T] & $1.35 \sim 1.41$ \\
& Length [mm] & 99.2 \\
& Width [mm] & 24.1 \\
& Thickness [mm] & 8.9 \\
\hline \multirow{5}{*}{ Armature (Stator) } & Length [mm] & 160 \\
& Width [mm] & 207 \\
& Thickness [mm] & 260 \\
& Slot number [mm] & 6 \\
\hline
\end{tabular}

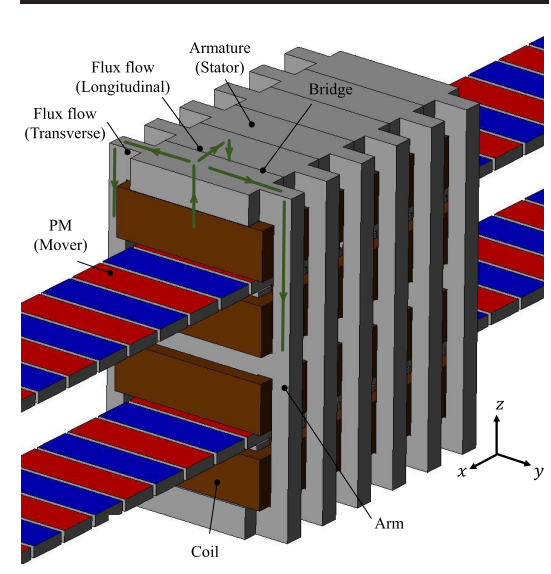

(a) Hybrid transverse-flux type PMLSM structure

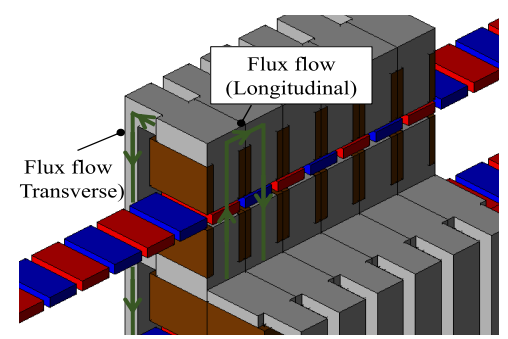

(b) Enlarged cut model showing the flow of longitudinal flux path inside armature

Fig. 1. Hybrid transverse-flux type PMLSM structure under investigation

magnetic flux flow switches to longitudinal direction. Thus, higher air gap flux is attainable without core saturation, ensuring a wide linear operating range.

With higher air gap flux densities, the unwanted detent force is also increased, leading us to the following section which introduces the proposed method for detent reduction.

\section{Detent Force Reduction by Mover Shifting}

3.1 Mover Shifting and Detent Force Since the twomover PMLSM can be treated as two independent PMLSMs stacked on top of each other, we consider the contribution of each side separately. The contribution to the detent force from the upper PM mover is $f_{d}^{u}(x)$, expressed as Fourier series owing to its periodic nature.

$$
f_{d}^{u}(x)=\sum_{n=2}^{N}\left(f_{c n} \cos \left(\frac{n \pi}{\tau_{p}} x\right)+f_{s n} \sin \left(\frac{n \pi}{\tau_{p}} x\right)\right) \cdots \cdots
$$

Where $f_{c n}$ and $f_{s n}$ are Fourier coefficients and $x$ is the position of the mover. Here, $n \in \mathcal{E}$, where $\mathcal{E}$ is a set of even numbers. By shifting the lower PM mover by $x_{0}$ along $x$ axis, the contribution to the detent force $f_{d}^{l}$ from lower PM 
side is calculated as:

$$
f_{d}^{l}(x)=f_{d}^{u}\left(x-x_{0}\right)
$$

Then the total detent force described as sum of the upper and lower movers is given as:

$$
F_{d}(x)=f_{d}^{u}(x)+f_{d}^{u}\left(x-x_{0}\right) \cdots \ldots \ldots \ldots \ldots \ldots \ldots \ldots \ldots \ldots \ldots
$$

In case when $x_{0}=0$, the detent force for the conventional case $F_{d 1}(x)$ is given as:

$$
F_{d 1}(x)=2 f_{d}^{u}(x)
$$

This is natural as both upper and lower contributions are exactly equal. In case when $x_{0}=\tau_{p} / 2$, the total detent force is calculated as:

$$
\begin{aligned}
F_{d 2}(x)= & \sum_{n=2}^{N} 2 \cos \left(\frac{n \pi}{4}\right)\left(f_{c n} \cos \left(\frac{n \pi}{\tau_{p}}\left(x-\frac{\tau_{p}}{4}\right)\right)\right. \\
& \left.+f_{s n} \sin \left(\frac{n \pi}{\tau_{p}}\left(x-\frac{\tau_{p}}{4}\right)\right)\right) \ldots \ldots \ldots \ldots
\end{aligned}
$$

As can be observed,

$$
\cos \left(\frac{n \pi}{4}\right)=\left\{\begin{array}{ll}
0 & n=2,6,10 \ldots 4 k-2 \\
(-1)^{n / 4} & n=4,8,12, \ldots 4 k
\end{array} .\right.
$$

Where $k \in \mathcal{N}$ natural numbers. This verifies that along with the fundamental harmonic $n=2$, all harmonics up to $4 k-2$ are ideally eliminated. The remaining most significant harmonic is $n=4$.

3.2 Mover Shifting and Thrust An intrinsic advantage of our machine is observed when phase of the lower PM mover is shifted by $\tau_{P} / 2$. The magnetic flux return path changes its direction as shown in Fig. 2 and Fig. 3. Under the assumption of no saturation, the total magnetic flux at both air gaps, remains unchanged. However, there does exist now a phase difference between upper and lower sides and with original coil phase arrangement, thrust would be reduced. Therefore, the phase sequence of lower side is re-adjusted.

3.2.1 Phase Sequence Re-adjustment We employ the phasor diagram to reassign the winding phase. Being a three phase AC machine, there are but only six ways a winding can be excited. As a result, the phasor diagram is divided into six equal sectors of $60 \mathrm{deg}$ each represented by dashed black lines. Each armature teeth is assigned a number starting from one, which is at the front. The motor moves along positive $x$-axis, however, starting from the first teeth, the remaining ones are arranged along negative $x$-axis with increasing number, which translates to a clockwise rotation on the phasor diagram. It should however be noted that since the relative phase between the teeth is important, starting position (angle) of the first teeth can be chosen arbitrarily. With physical distance between each teeth equal to one slot-pitch $\tau_{S}$, it translates to:

$$
\tau_{s}=\frac{N_{p} \tau_{P}}{N_{s}} \rightarrow \theta_{\tau_{S}}=\frac{N_{p} \pi}{N_{s}}
$$

Where $\theta_{\tau_{S}}$ is the electrical angle corresponding to $\tau_{S}$. With consecutive clockwise rotation corresponding to teeth-teeth electrical angle, each teeth is assigned its respective location

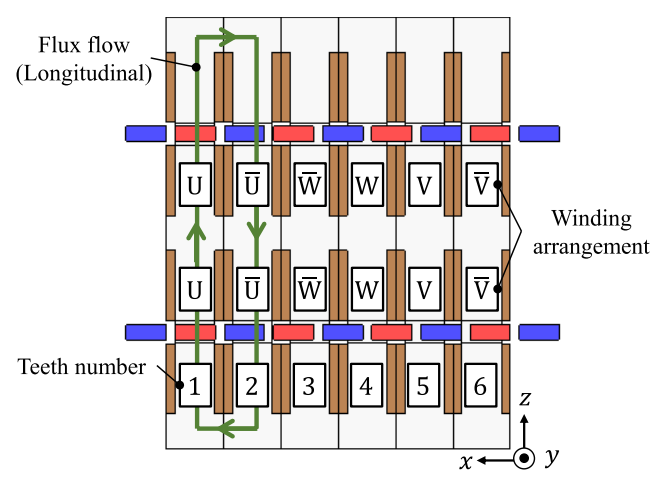

(a) Conventional (No phase shift between PM sides)

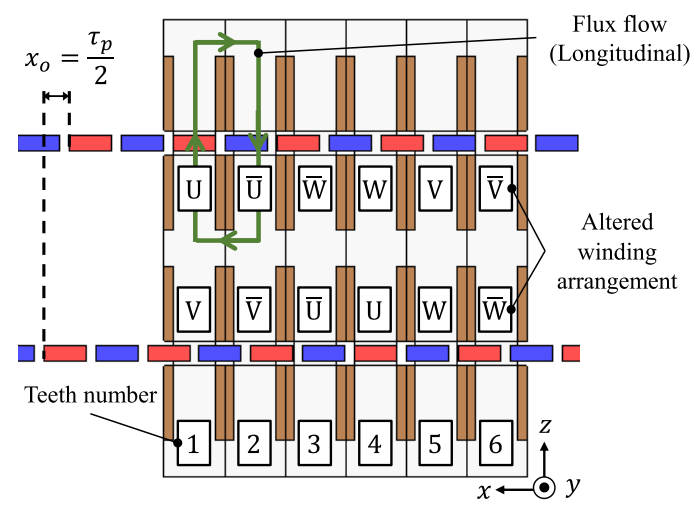

(b) Proposed (A phase shift of $\frac{\tau_{P}}{2}$ between upper and lower PM sides)

Fig. 2. Magnetic flux path and winding arrangement of PMLSM under consideration (Side views)

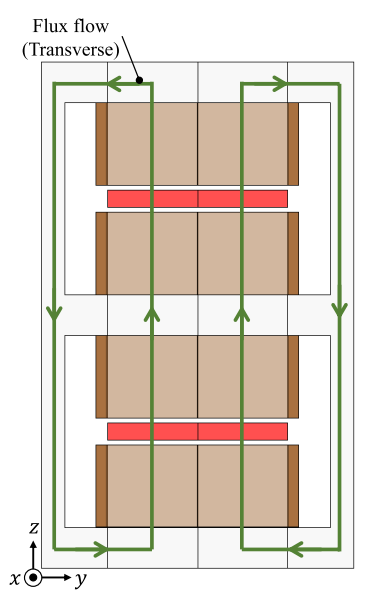

(a) Conventional

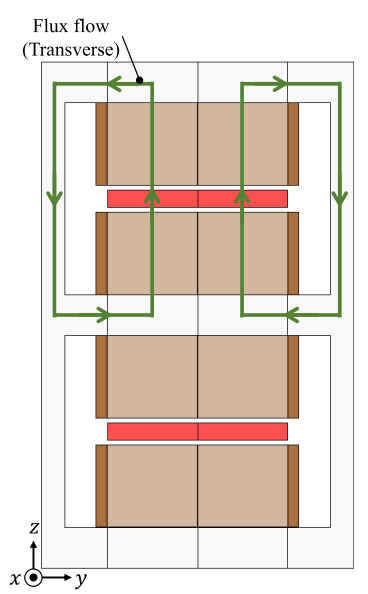

(b) Proposed
Fig. 3. Magnetic flux path of PMLSM under consideration (Front views)

on the diagram. The excitation phase is then assigned to each teeth depending on which sector it lies in.

For instance, consider the phasor diagram of $6 \tau_{S}=7 \tau_{P}$ configuration shown in Fig. 4(a). The first teeth is assigned a numbered (1) colored line aligned with zero degrees. Slot angle $\theta_{\tau_{S}}=210^{\circ}$ and thus the colored line, representing teeth 2 , falls in angular sector in between $150^{\circ} \leq \theta_{\tau_{S}}<210^{\circ}$, and thus is assigned the phase -U. From teeth 2 onwards, the same procedure is repeated consecutively for adjacent teeth and the phase location of each teeth is found.

Following the above explained method, the phase sequence of the lower side is obtained as shown in Table 2 and as illustrated in Fig. 4(d). The difference between Fig. 4(a) and 


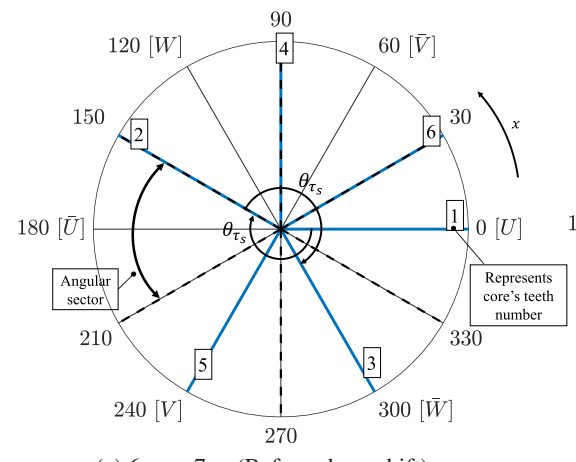

(a) $6 \tau_{S}=7 \tau_{P}$ (Before phase shift)

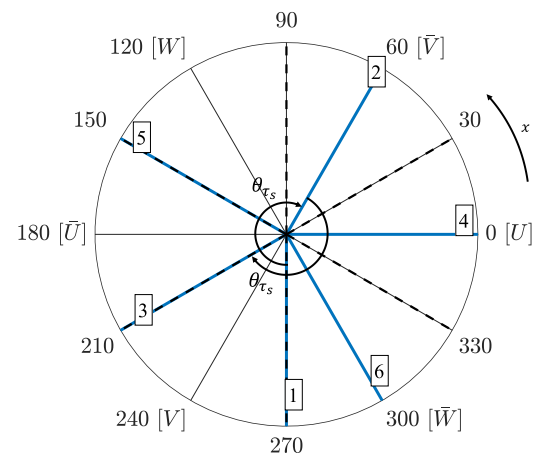

(d) $6 \tau_{S}=7 \tau_{P}$ (After phase shft)

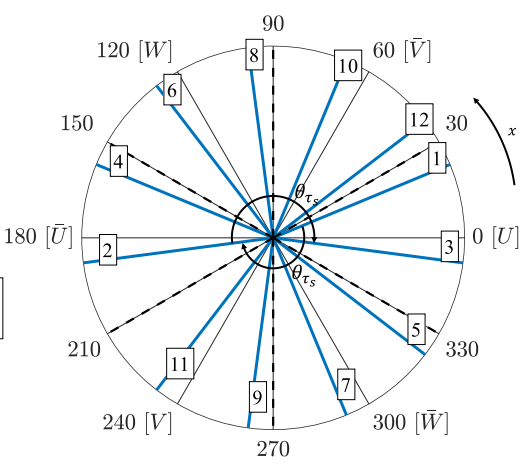

(b) $12 \tau_{S}=13 \tau_{P}$ (Before phase shift)

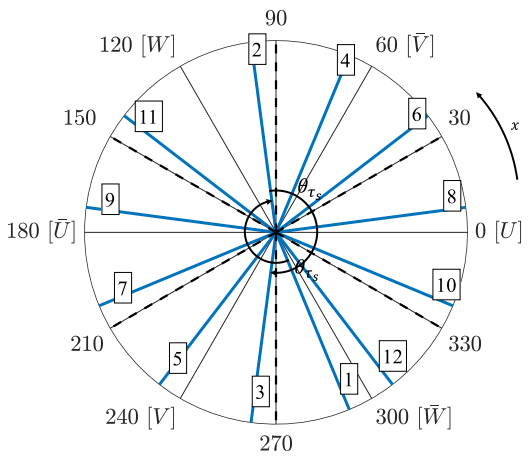

(e) $12 \tau_{S}=13 \tau_{P}$ (After phase shft)

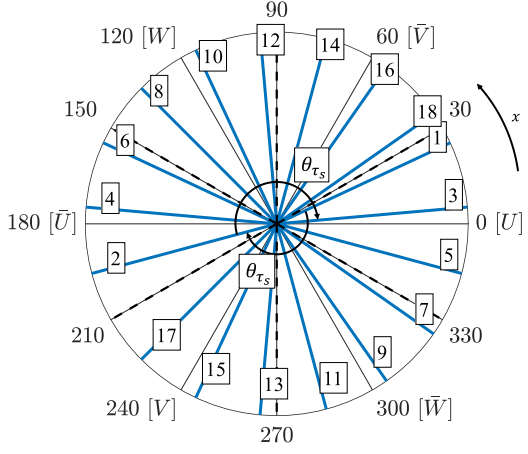

(c) $18 \tau_{S}=19 \tau_{P}$ (Before phase shift)

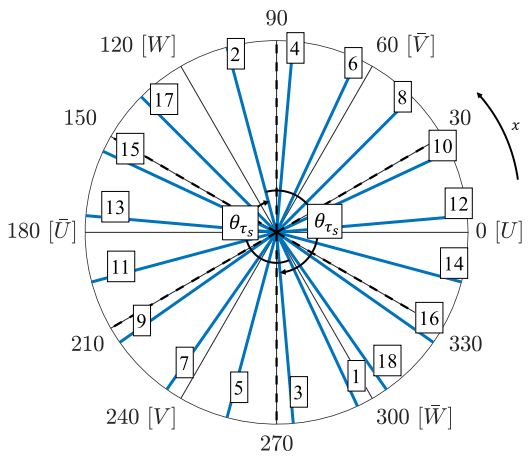

(f) $18 \tau_{S}=19 \tau_{P}$ (After phase shft)

Fig. 4. Phase arrangement of armature teeth for different applicable pole-slot combinations

Table 2. Electric degree and phase of amarture slot

\begin{tabular}{c|c|c|c|c}
\hline & \multicolumn{2}{|c|}{ Conventional (Before shift) } & \multicolumn{2}{|c}{ Proposed (After shift) } \\
\hline Slot No. & Electrical degree & Coil phase & Electrical degree & Coil phase \\
\hline 1 & $0^{\circ}$ & $\mathrm{U}$ & $270^{\circ}\left(-90^{\circ}\right)$ & $\mathrm{V}$ \\
2 & $150(-210)^{\circ}$ & $-\mathrm{U}$ & $60^{\circ}$ & $-\mathrm{V}$ \\
3 & $300^{\circ}$ & $-\mathrm{W}$ & $210^{\circ}$ & $-\mathrm{U}$ \\
4 & $90^{\circ}$ & $\mathrm{W}$ & $0^{\circ}$ & $\mathrm{U}$ \\
5 & $240^{\circ}$ & $\mathrm{V}$ & $150^{\circ}$ & $\mathrm{W}$ \\
6 & $30^{\circ}$ & $-\mathrm{V}$ & $300^{\circ}$ & $-\mathrm{W}$ \\
\hline
\end{tabular}

Figure 4(d) can be observed in the starting position of the numbered colored line 1 , representing the first teeth of lower $\mathrm{PM}$ side which has been shifted by $90^{\circ}$. The consequence of this phase readjustment is that the original thrust is recovered. This is achieved without any major alteration to the armature's mechanical structure except changes in winding connections affirming ease of applicability, usefulness of this method and widespread practicality of our machine.

It is pertinent is mention here that this method's applicability depends on fractional slot-pole structures. For a fundamental three phase unit such as $3 \tau_{S}=2 \tau_{P}$ or $3 \tau_{S}=4 \tau_{P}$, application of phase shift would result in thrust reduction. Contrarily, fractional high slot-pole combination provides an added freedom of choice for winding phase since more coverage of entire period ( $360 \mathrm{deg}$ ) is available. And thus, any phase difference between armature and field side PM poles can be accommodated by a simple coil phase re-arrangement.

3.3 Applicable Slot-pole Structures Although, the method is applied to a $6 \tau_{S}=7 \tau_{P}$ structure, here we provide the conditions for slot-pole combination of our machine to which this method is applicable. First, the following preconditions are set:

(1) Being a three phase machine, the number of slots $N_{s}$ is a multiple of three i.e $N_{s}=3 n_{s}$.

(2) The number of poles $N_{p}=N s \pm 1$.

Where $n_{s} \in N$ natural numbers. Number of slots and the number of poles are also related to each other as:

$$
N_{s} \cdot \tau_{s}=N_{p} \cdot \tau_{p} \cdot
$$

Detent force is reduced by shifting the lower mover by an electrical angle of $\pi / 2$. However, to prevent thrust reduction, a necessary condition for the pole-slot combination to work is that there must exist an armature pole (teeth) which lies exactly $\pi / 2 \operatorname{rad}$ or $3 \pi / 2 \mathrm{rad}$ away from the first armature pole (teeth). If this condition is satisfied, phase shifting of PM mover by $\pi / 2$ rad does not add additional phase difference between armature teeth and the exact UVW axis (See Fig. 4). We formulate this condition as follows:

$$
\bmod \left(\pi\left(N_{n}-1\right) \frac{N_{p}}{N_{s}}, 2 \pi\right)=\frac{\pi}{2} \text { or } \frac{3 \pi}{2} \ldots \ldots \ldots \ldots
$$

Here, mod represents the modulo operation. Where $N_{n}$ satisfies the constraints $1 \leq N_{n} \leq 3 n_{s}$ and represents the armature teeth number which is exactly $\pi / 2$ or $3 \pi / 2$ rad away from the first teeth. For any value of $N_{n}$ and $n_{s}$, the above condition can be restated as:

$$
\bmod \left(\left(N_{n}-1\right) \frac{3 n_{s} \pm 1}{3 n_{s}}, 2\right)=0.5 \text { or } 1.5 \cdots \cdots \cdots
$$

If the modulus of the expression inside the brackets equals 0.5 or 1.5 , it means that there does exists an armature teeth exactly $\pi / 2$ or $3 \pi / 2$ rad away from first one and for the then calculated value of $N_{n}$, this method is applicable on the obtained the pole-slot combination $\left(N_{s}, N_{p}\right)$. Table 3 provides a 
Detent Reduction by Positional Shifting of Permanent Magets for a PMLSM Without Compromising Thrust (Yuto Mitsui et al.)

Table 3. Applicable slot-pole combinations calculated by solving (10) for $N_{n}$

\begin{tabular}{|c|c|c|c|c|}
\hline$n_{s}$ & $1 \leq N_{n} \leq 3 n_{s}$ & Eq (10) & $\left|N_{s}\right|$ & $N_{p}$ \\
\hline \multirow{3}{*}{2} & \multirow{3}{*}{$(1,2,3, \underline{4}, 5,6)$} & $(0,1.33,0.66)$ & & \\
\hline & & $0,1.16,0.33, \underline{\mathbf{1 . 5}}, 0.66,1.83$ & 6 & 5 \\
\hline & & $(0,0.16,0.33, \underline{\mathbf{0 . 5}}, 0.66,0.83)$ & 6 & 7 \\
\hline 3 & $(1,2,3,4,5,6,7,8,9)$ & $(0,1.11,0.22,1.33,0.44,1.55,0.66,1.77,0.88)$ & & \\
\hline \multirow{2}{*}{4} & \multirow{2}{*}{$(1,2,3,4,5,6, \underline{7}, 8,9,10,11,12)$} & $(0,1.92,1.83,1.75,1.67,1.58, \underline{\mathbf{1 . 5 0}}, 1.42,1.33,1.25,1.17,1.08)$, & 12 & 11 \\
\hline & & $(0,0.08,0.17,0.25,0.33,0.42, \underline{\underline{\mathbf{0 . 5}}}, 0.58,0.67,0.75,0.83,0.92)$, & 12 & 13 \\
\hline \multirow[t]{3}{*}{5} & \multirow{3}{*}{$\begin{array}{c}(1,2,3,4,5,6,7,8,9,10,11,12,13,14,15) \\
(1,2,3,4,5,6,7,8,9, \underline{\mathbf{1 0}}, 11,12,13,14,15,16,17,18)\end{array}$} & $(0,1.06,0.13,1.2,0.26,1.33,0.4,1.46,0.53,1.6,0.66,1.73,0.8,1.86,0.93)$ & & \\
\hline & & $(0,1.94,1.89,1.83,1.78,1.72,1.67,1.61,1.56, \underline{\mathbf{1 . 5 0}}, 1.44,1.39,1.33,1.28,1.22,1.17,1.11,1.06)$ & 18 & 17 \\
\hline & & $(0,0.06,0.11,0.17,0.22,0.28,0.33,0.39,0.44, \mathbf{0 . 5 0}, 0.56,0.61,0.67,0.72,0.78,0.83,0.89,0.94)$ & 18 & 19 \\
\hline
\end{tabular}

Table 4. Detent reduction for different pole-pair configurations

\begin{tabular}{c|c|c|c|c}
\hline \multirow{2}{*}{$\begin{array}{c}\text { Pole-pair } \\
\text { configuration }\end{array}$} & \multicolumn{2}{|c|}{ Before phase shift } & \multicolumn{2}{|c}{ After phase shift } \\
\cline { 2 - 5 } & $F_{\text {det }}[\mathrm{N}]$ & $F_{x}[\mathrm{~N}]$ & $F_{\text {det }}[\mathrm{N}]$ & $F_{x}[\mathrm{~N}]$ \\
\hline $6 \tau_{s}=7 \tau_{p}(\operatorname{Exp})$ & $149.7(1)$ & - & $24.3(0.16)$ & - \\
$13 \tau_{s}=12 \tau_{p}$ & $157.8(1)$ & $3357(1)$ & $18.6(0.11)$ & $3298(0.98)$ \\
$18 \tau_{s}=19 \tau_{p}$ & $180.6(1)$ & $4967(1)$ & $35.3(0.19)$ & $5019(1.01)$ \\
\hline
\end{tabular}

list of applicable values of slot-pole combination $N_{s}$ and $N_{p}$ for which the proposed detent reduction method with thrust retainment can be applied. These are calculated by solving (10) for $N_{n}$. For $n_{s}=\{1,2,3,4,5,6\}$, the obtained values of $N_{n}$ (underlined) which satisfy (10) are given. For instance, when $n_{s}=1$, there exists no value of $N_{n}$ which satisfies (10). On the other hand, when $n_{s}=2$, there does exist a value of $N_{n}=4$ (underlined), for which (10) equals 1.5 and 0.5 for the case when $N_{p}=N_{s}-1$ and $N_{p}=N_{s}+1$, respectively, giving the applicable slot-pole configuration. We observe that this results in $N_{s}$ being multiples of six.

3.4 Feasibility of Proposed Method on Other Poleslot Configurations To substantiate the usefulness of the method, it is applied on the different slot-pole configurations obtained from Table 3 which are $6 \tau_{s}=7 \tau_{p}, 12 \tau_{s}=13 \tau_{p}$ and $18 \tau_{s}=19 \tau_{p}$. Each teeth is numbered and its appropriate phase sequence is obtained from phasor diagrams shown in Fig. 4. With increasing number of poles, it is observed that the entire 360 deg of electrical angle is densely covered by the teeth locations, having appropriate excitation. All the numerical analyses in this study are performed by finite element method using electromagnetic field analysis software called JMAG. The results, before (conventional) and after (proposed) phase shifting are shown in Fig. 5. As expected, for the later two cases, the second harmonic is eliminated, leaving the fourth harmonic as the most dominant one with amplitudes $18.6[\mathrm{~N}]$ and $35.3[\mathrm{~N}]$, respectively. Contrary to detent, there is almost no change in the thrust of the motor (See Table 4). For $6 \tau_{s}=7 \tau_{p}$, the numerical simulation results as well as measured data are shown later in the next section, along with experimental verification on a prototype.

\section{Experimental Verification}

4.1 Prototype Assembly To confirm the experimental validity of the proposed method, a prototype of $6 \tau_{s}=7 \tau_{p}$ configuration, shown in Fig. 6 is manufactured. The assembly of the said machine is simple. Two distinct shaped laminated sheets are prepared. Part of the armature which forms

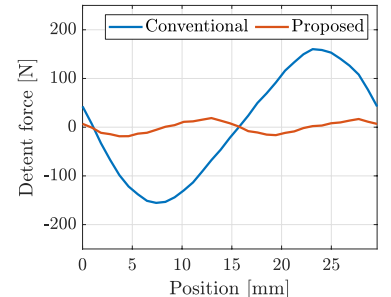

(a) Detent waveform $\left(12 \tau_{s}=13 \tau_{p}\right)$

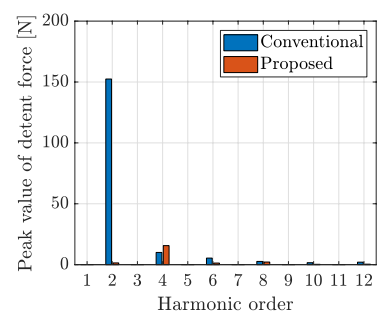

(c) Detent harmonic analysis $\left(12 \tau_{s}=13 \tau_{p}\right)$

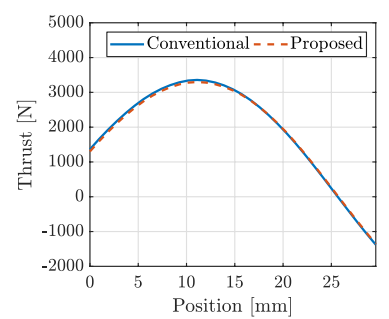

(e) Static thrust waveform $\left(12 \tau_{s}=13 \tau_{p}\right)$

(f) Static thrust waveform $\left(18 \tau_{s}=19 \tau_{p}\right)$

Fig. 5. 3D-FEM Simulation results for conventional and proposed configuration of $12 \tau_{s}=13 \tau_{p}$ and $18 \tau_{s}=19 \tau_{p}$ slot-pole combination:

the teeth as well as the arm region is assembled by stacking the laminated sheets, shaped in the form as shown in Fig. 6(a), along x-axis. Wound coils are then inserted over each teeth.

The bridge structure is composed of rectangular shaped laminated sheets which are placed in-between each armature teeth (see Fig. 6(b)) at three locations, top, center and bottom (see Fig. 6(b)). Although it is unconventional to place laminated sheets perpendicular to flux flow, it was found that as the arm region saturates, the magnetic flux flow changes in a continuous manner from transverse flux to longitudinal flux through this bridge region. This allowed increased magnetic field near the air gap, resulting in higher thrust densities. Finally. bolts through entire body of stacked sheets are inserted and tightened at both ends.

4.2 Measurement Setup In order to measure detent 
and thrust waveforms, the mover (PMs) is connected to a load cell as shown in Fig. 6(c). Under no armature excitation, by traversing along $\mathrm{x}$-axis and simultaneously logging position and load cell data, the required detent waveforms are recorded. Similarly, under DC armature excitation, static thrust waveform is measured, from which the maximum value which corresponds the three phase constant thrust is plot against DC armature current. The details are given in section 4.4.

Experimental data obtained is for two configurations for comparison.

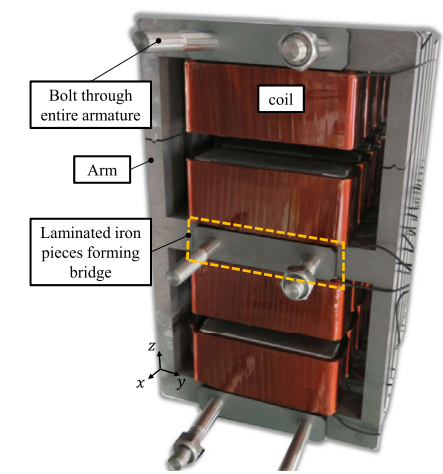

(a) Front view (PM mover not shown)

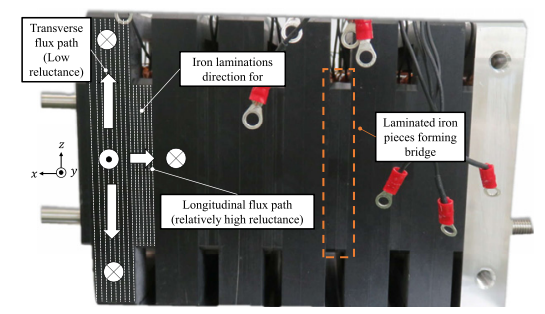

(b) Top view

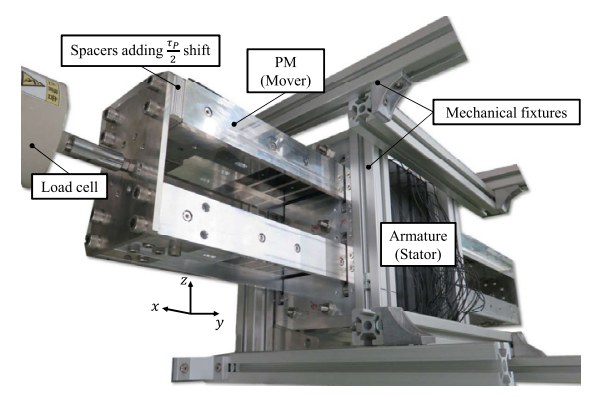

(c) Detent and thrust measurement setup

Fig. 6. Manufactured prototype machine
(1) Conventional configuration where there is no phase shift between the upper and lower PM sides. Winding configuration used is that shown in the Table 2.

(2) Proposed configuration where a phase shift of $\frac{\tau}{2}$ is introduced by addition of spacers in one of the PM sides, while simultaneously re-adjusting the coil end connections to match the armature excitation sequence shown in Table 2.

4.3 Detent Force Comparison Measured detent force waveform of both, conventional and proposed configuration are shown in Fig. 7(a). Compared to conventional case, a reduction of $83.7 \%$ from a peak value of 149.7 [N] to $24.3[\mathrm{~N}]$ is achieved. Despite having approximately equal peak values, a slight difference in harmonic content is observed between experimental and simulated (3D-FEM) waveforms. This is corroborated by comparing the harmonic analysis of each set of data as shown in Fig. 7(b) and Fig. 7(c).

This dissimilarity can be denoted to the fact that since detent force is highly sensitive to relative positional arrangement of teeth, even a slight deviation, which may have been caused by mechanical tolerances may give rise to such differences in higher harmonics. Nonetheless, the authors would like to emphasize that the focus of this paper is on the drastic reduction of detent force at the cost of minimal mechanical alteration and not on the comparison between experimental and simulated results, which is outside the scope of this paper.

Continuing the discussion on the results, as per Eq. (5), harmonics of order $n=2,6,10$ are canceled out. Being a $6 \tau_{S}=7 \tau_{P}$ structure, the slot-effect in the 12th harmonic is evident in the results. Although, harmonic analysis shows orders of $n=3,5,7 \ldots$ which ideally should not exist for a detent force. These are a by product due to noise in measurement and are disregarded in our discussion.

4.4 Thrust Comparison Single-phase measured thrust characteristics for both, conventional and proposed, configurations are shown in the Fig. 8 when DC current is applied to only one phase. Motivation behind this step is to experimentally validate the claim that, despite phase shift which was required for detent reduction, re-adjustment of coils ensured no loss of thrust for individual phases. For Uphase and V-phase, there is negligible difference. However, for $\mathrm{W}$-phase, the thrust reduces slightly as armature excitation is increased. This is attributed to the fact that in conventional case, the $\mathrm{W}$-phase is sandwiched between the other two phases resulting in a slightly stronger magnetic flux link-

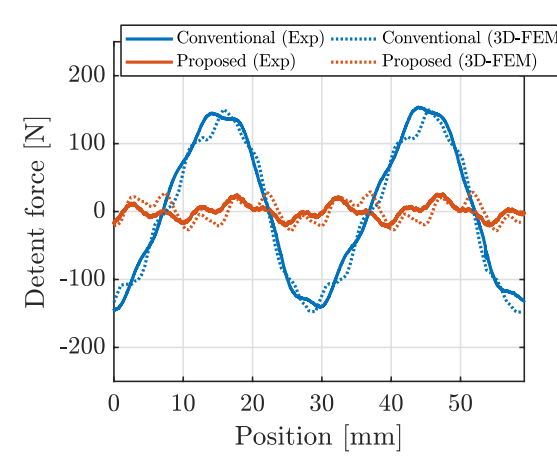

(a) Detent force waveforms

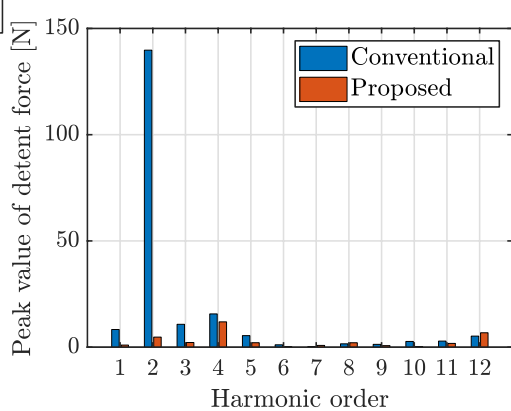

(b) Harmonic analysis of measured detent force

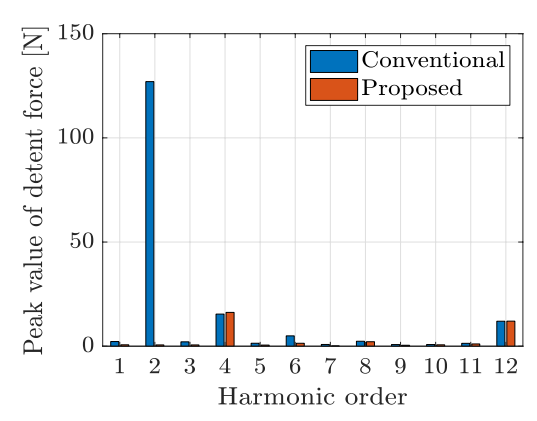

(c) Harmonic analysis of simulated (JMAG) detent force

Fig. 7. Comparison of detent force between conventional and proposed configurations 


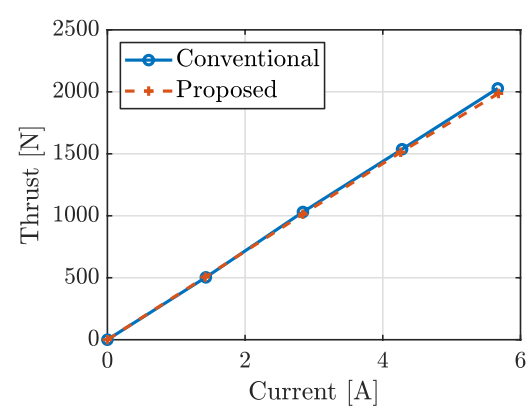

(a) U-phase

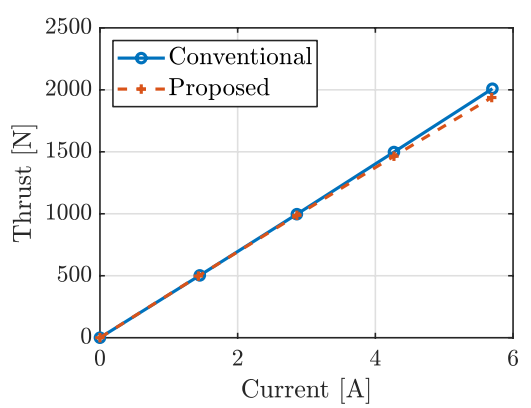

(b) V-phase

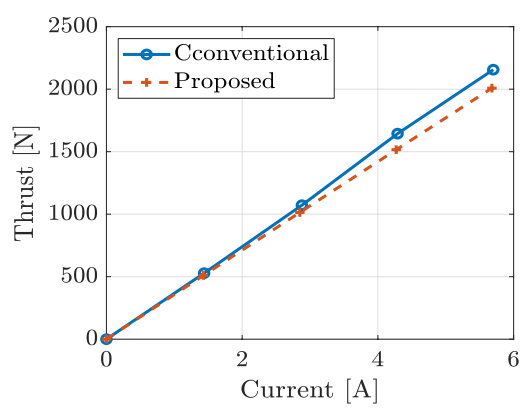

(c) W-phase

Fig. 8. Measured thrust for single phase against DC excitation current

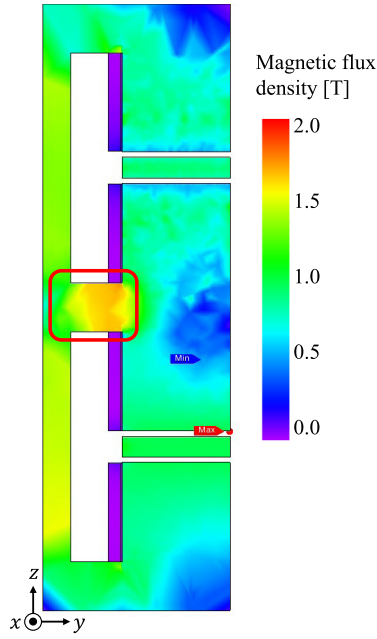

Fig. 9. Magnetic flux density of prototype (1/2 model)

age. Furthermore, as long as armature's saturation is insignificant, the electromotive force (EMF) and consequently the thrust, before and after phase shifting does not change. However, at higher excitation, the common in-between section, highlighted in red box in Fig. 9, which provided a bypass to magnetic flux after phase shifting saturates and thus acts as a bottleneck for magnetic flux, resulting in slight reduction in thrust.

Assuming that the single-phase current is $I_{1}$ and the thrust constant is $K_{t}$, the following equation holds for the singlephase thrust $F_{t 1}$.

$$
F_{t 1}=K_{t} I_{1}
$$

Also, assuming that the three-phase current is $I_{3}$, the following equation holds for the three-phase thrust $F_{t 3}$.

$$
\begin{aligned}
F_{t 3} & =K_{t} I_{3}+\frac{1}{2} K_{t} I_{3} \cos \left(60^{\circ}\right)+\frac{1}{2} K_{t} I_{3} \cos \left(60^{\circ}\right) \\
& =K_{t} \times \frac{3}{2} I_{3} \ldots \ldots \ldots \ldots \ldots \ldots \ldots \ldots \ldots \ldots \ldots \ldots \ldots \ldots \ldots \ldots \ldots \ldots \ldots \ldots \ldots \ldots \ldots \ldots \ldots \ldots \ldots \ldots \ldots \ldots \ldots \ldots \ldots \ldots \ldots \ldots
\end{aligned}
$$

In order to generate equal amount of three phase thrust using single phase excitation, the following relation can be used.

$$
I_{1}=\frac{3}{2} I_{3}
$$

Single and three phase thrust normalized to excitation current according to Eq. (13) is shown in Fig. 10. The motor constant $K_{t}$ calculated is $350 \mathrm{~N} / \mathrm{A}$. It is confirmed that there is no difference in magnitude between the three-phase thrust and the

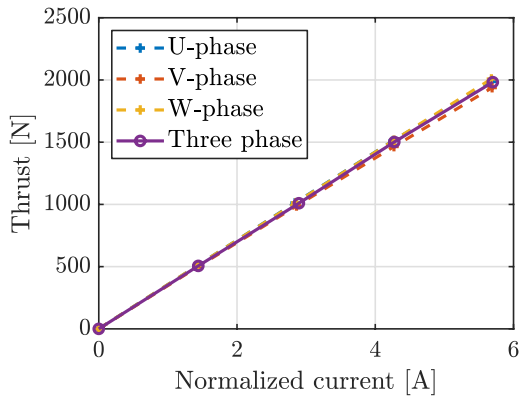

Fig. 10. Measured single phase and three phase thrust

single-phase thrust, validating the assumption that each phase of upper and lower sections, behaves independently, despite being a single core. This allowed us to reassign the phase sequence of the lower section along with the shifting of PM, and achieving detent reduction with thrust retainment.

\section{Conclusion}

A useful yet simple method for reducing the unwated detent force was proposed and its ease of applicability was verified by experimental results. The detent's peak was reduced down to $24.3[\mathrm{~N}]$ from 149.7 [N], a drastic improvement with minimal structural alteration. Despite being a single core, the thrust was maintained with a simple reassignment of phases, which translates to no additional cost, emphasizing the practicality of the proposed method. It was also shown that under the proposed phase shift, each phase behaved with minimal interaction with the other, despite being magnetically connected. Apart from the manufactured prototype, a general formulation for applicable slot-pole configuration was also given along with verification on two other pole-slot structures. Both showed similar reduction in peak detent forces without compromising thrust force. In future, the authors plan to consider further reduction of second most dominant harmonic, the fourth one.

\section{References}

( 1 ) Z.J. Piech J.F. Gieras, and B. Tomczuk: Linear Synchronous Motors: Transportation and Automation Systems, 2nd ed. CRC Press.

( 2 ) C.C. Hwang, M.H. Wu, and S.P. Cheng: "Influence of pole and slot combinations on cogging torque in fractional slot $\{\mathrm{PM}\}$ motors", Journal of Magnetism and Magnetic Materials, Vol.304, No.1, pp.430-432 (2006)

( 3 ) Z.Q. Zhu and D. Howe: "Influence of design parameters on cogging torque in permanent magnet machines", IEEE Transactions on Energy Conversion, Vol.15, No.4, pp.407-412 (2000)

( 4 ) Z.Q. Zhu, S. Ruangsinchaiwanich, and D. Howe: "Synthesis of coggingtorque waveform from analysis of a single stator slot", IEEE Transactions on 
Industry Applications, Vol.42, No.3, pp.650-657 (2006)

( 5 ) S. Ruangsinchaiwanich, Z.Q. Zhu, and D. Howe: "Influence of magnet shape on cogging torque and back-emf waveform in permanent magnet machines", pp.284-289 (2008)

( 6 ) J.S. Shin, R. Watanabe, T. Koseki, H.J. Kim, and Y. Takada: "The design for cogging force reduction of a double-sided transverse flux permanent magnet linear synchronous motor", IEEE Transactions on Magnetics, Vol.50, No.11, pp.1-4 (2014)

( 7 ) Y.w. Zhu, S.G. Lee, K.S. Chung, and Y.H. Cho: "Investigation of auxiliary poles design criteria on reduction of end effect of detent force for pmlsm", IEEE Transactions on Magnetics, Vol.45, No.6, pp.2863-2866 (2009)

( 8 ) C.C. Hwang, P.L. Li, and C.T. Liu: "Optimal design of a permanent magnet linear synchronous motor with low cogging force", IEEE Transactions on Magnetics, Vol.48, No.2, pp.1039-1042 (2012)

( 9 ) H. Zhang, B. Kou, Y. Jin, and H. Zhang: "Investigation of auxiliary poles optimal design on reduction of end effect detent force for pmlsm with typical slot pole combinations", IEEE Transactions on Magnetics, Vol.51, No.11, pp.1-4 (2015)

(10) Z.P. Xi and D. Howe: "Redluction of cogging force in slotless linear per im a n e n", IEE Proceedings - Electric Power Applications, Vol.144 (1997)

(11) M. Inoue and K. Sato: "An Approach to a Suitable Stator Length for Minimizing the Detent Force of Permanent Magnet Linear Synchronous Motors", IEEE Transactions on Magnetics, Vol.36, 4 PART 1, pp.1886-1889 (2000)

(12) Y.S. Kwon and W.j. Kim: "Detent-force minimization of double-sided interior permanent-magnet flat linear brushless motor", IEEE Transactions on Magnetics, Vol.52, No.4, pp.1-9 (2016)

(13) S. Ahmed, T. Koseki, and H.J. Kim: "Detent force reduction for a novel transverse flux permanent magnet linear synchronous motor without compromising stroke length", LDIA 2017 - 11th International Symposium on Linear Drives for Industry Applications (2017)

(14) Y. Aoyama, Y. Hasegawa, M. Sasaki, J. Nakatsugawa, Y. Iwaji, and A. Komura: "Development of high-acceleration linear motor to realize resource saving and high productivity", IEEJ Transactions on Industry Applications, Vol.137, No.4, pp.295-301 (2017)

Yuto Mitsui (Non-member) completed his Bachelors degree in 2018

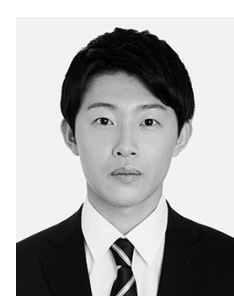

Salman Ahmed (Student Member) received his Masters and Doctoral degree in Electrical Engineering in 2016 and 2019, respectively, from The University of Tokyo where he worked on optimal design of permanent magnet linear motors and control of magnetically levitated stages. Currently, he is an R\&D engineer at Fuji Electric Co., Ltd where he is engaged in development of electric railway propulsion systems. He is also a student member of International Electrical and Electronics Engineers.

Yasuaki Aoyama (Member) completed his Masters degree from

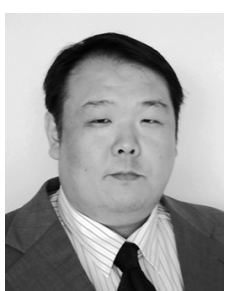
Graduate School of Science and Technology, Tokyo University of Science in March 1998. In April same year, He joined Shin-Etsu Chemical Co., Ltd. Later, he joined Hitachi, Ltd. in February 2007. Currently, he is working for Control Innovation Center, Research and Development Group and is a Chief Researcher at the center. He is engaged mainly in R\&D of motor and drive system.

Takafumi Koseki (Member) received the Ph.D. degree in electrical

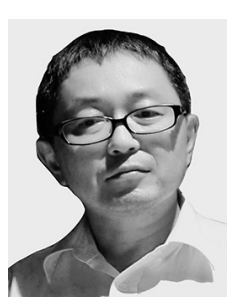
engineering from The University of Tokyo, Tokyo, Japan, in 1992. He is currently a Professor with the Department of Electrical Engineering, School of Engineering, The University of Tokyo. His current research interests include public transport systems, particularly linear drives, and the analysis and control of traction systems. Prof. Koseki is a member of the Institute of Electrical Engineers of Japan, Japan Society of Mechanical Engineering, Japan Society of Applied Electromagnetics and Mechanics, Japan Society of Precision Engineering, and Japan Railway Electrical Engineering Association. 